\title{
Variations
}

Variations

Revue internationale de théorie critique

$21 \mid 2018$

L'industrie de la culture : version originale

\section{Mobilité, hybridité, liquidité : un architexte de la globalisation?}

\section{Marc Bernardot}

\section{(2) OpenEdition}

Journals

Édition électronique

URL : http://journals.openedition.org/variations/937

DOI : 10.4000/variations.937

ISSN : 1968-3960

Éditeur

Les amis de Variations

Référence électronique

Marc Bernardot, « Mobilité, hybridité, liquidité : un architexte de la globalisation ? », Variations [En ligne], 21 | 2018, mis en ligne le 05 avril 2018, consulté le 22 avril 2019. URL : http://journals.openedition.org/ variations/937 ; DOl : 10.4000/variations.937

Ce document a été généré automatiquement le 22 avril 2019

Les ami•e•s de Variations 


\title{
Mobilité, hybridité, liquidité : un architexte de la globalisation?
}

\author{
Marc Bernardot
}

1 Les mutations accélérées de la période contemporaine sont nommées de manière maintenant standardisée comme une globalisation ${ }^{1}$ ou en français une mondialisation ${ }^{2}$. Celle-ci peut être sommairement décrite comme la mise en mouvement accélérée des individus, des biens et des informations (migrations, commerce, numérisation...). Elle est aussi parfois associée aux révolutions urbaines, bancaires, de la robotique et de la génétique, à la remise en question des Etats traditionnels, des frontières et l'émergence d'un village mondial cosmopolite. Elle est enfin rendue responsable de l'accroissement des risques de conflits, de catastrophe écologique mondiale, voire de remplacement ou d'extinction des espèces vivantes.

2 Je propose dans cet article d'étudier trois notions utilisées pour traduire en mots, en récits et en images ces processus de transformations accélérées des formes sociales, culturelles et politiques en mettant en évidence à la fois leur potentiel heuristique et les contraintes liées à leurs usages métaphoriques pour comprendre le monde contemporain. En effet la mobilité, l'hybridité et la liquidité sont utilisées de manière croissante dans les sciences humaines et sociales et les sciences en général, mais aussi dans les langages populaires, les lexiques experts et les discours médiatiques et politiques, pour caractériser des tendances à la remise en question des catégories et des institutions emblématiques de la modernité. La mobilité remet en cause le lieu, la position, le statut. L'hybridité questionne, transforme et supplante la race, l'identité, la norme. La liquidité menace la stabilité, la permanence et l'existence même de la réalité connue. De ce fait elles peuvent être envisagées chacune et ensemble, comme caractéristiques de la période actuelle qu'elle soit nommée postmodernité, modernité tardive, late capitalism, seconde modernité ou liquid modernity.

Il est possible de distinguer rapidement trois types de langages dans lesquels ces champs sémantiques sont employés. Au niveau le plus élémentaire, il existe des termes courants ou techniques pour décrire des phénomènes, des objets, des fonctions en lien avec ce qui se déplace, qui change d'état ou de forme. Il y a ensuite des discours imagés et des 
schémas narratifs dans les langages populaires, pédagogiques, économiques et politiques notamment, qui empruntent à ces lexiques pour rendre compte de manière simplifiée et "parlante » de phénomènes complexes ou intangibles et qui servent de grilles de compréhension, d'interprétation et de jugement. Il y a enfin des langues scientifiques qui recourent aux deux premiers registres pour constituer des modèles théoriques métaphoriques avec cette fois, accolée à l'objectif d'intelligibilité, une contrainte de cohérence des inférences ${ }^{3}$.

4 La méthode que j'ai suivie consiste d'abord à repérer des occurrences de ces items dans différents univers disciplinaires et cognitifs (sociologie, anthropologie, géographie, économie, droit, notamment). Les références présentées ici sont indicatives et je me permets de renvoyer à mes travaux récents sur ces questions pour des compléments bibliographiques ${ }^{4}$ tout en gardant à l'esprit le caractère exploratoire de ce travail dans un corpus lui-même globalisé et proliférant. Le matériel empirique et esthétique reste en arrière-plan. Il est le produit d'enquêtes de terrain de longue traine sur des thèmes corrélés en collaboration avec des équipes de recherche ces dernières années, (habitats non ordinaires, rapports à l'eau, sociologie de la recherche et de l'édition scientifique, mouvements sociaux, migrations, politiques publiques, art contemporain).

5 J'en viens à la méthode suivie pour le cheminement et le travail théoriques. D'un point de vue sémantique et sémiologique les difficultés sont multiples car, au-delà de la variété de leurs emplois, ces termes (je laisse ici de côté les images) décrivent à la fois des états et des processus (mobilisation, hybridation, liquidation par exemple, qui partagent le sens d'un auto-engendrement potentiel). De plus, leurs sens sont dynamiques et bien souvent tiraillés, c'est dans l'habitude des métaphores, entre des pôles antagoniques et en tension -y compris dans les acceptions ordinaires et les significations de base qui s'avèrent être, à premier abord au moins, des couples impossibles ou difficilement conciliables, par exemple la vitesse et la position, l'adaptation et la subversion, la clarté et l'imprécision, la pureté et la souillure, la fécondité et la corruption, etc. Ces différentes caractéristiques ne sont pas combinées en même temps et elles n'ont pas toutes la même valence. Les significations de ces mots-éponges ${ }^{5}$ peuvent se superposer, se mélanger ou se dissocier ${ }^{6}$. Selon la qualité des composants, leur quantité et leur vitesse de transformation, l'identité des acteurs qui les portent, leurs effets sont changeants.

6 J'utilise pourtant de cette tension et de cette variété pour en déterminer les pôles structurants dans un dispositif à la fois synchronique et diachronique, synthétique et analytique. Car ces termes et leurs transpositions métaphoriques sont, tout comme la potion de J. Derrida ${ }^{7}$ ou la sève de $\mathrm{P}$. Camporesi ${ }^{8}$, un Pharmakon à la fois remède et poison, tantôt guérisseur, tantôt mortel. Mon option est de rapprocher des thématiques a priori distantes les unes des autres mais qui se disent avec des mots et des images identiques. Je fais l'hypothèse que cela ouvre des possibilités d'une part de ré-investiguer les champs et les problématiques par-delà les frontières disciplinaires et d'autre part de proposer un point de départ d'une nouvelle cartographie dynamique de la pensée critique en sciences humaines et sociales posant les repères d'une langue transdisciplinaire partagée à partir d'observations multi-situées'.

7 Ces termes sont particulièrement employés dans les parlers et les lexiques comme des transpositions et des métaphores analogiques pour rendre compte d'un changement de forme, d'état et de position. Je formule l'hypothèse que leurs champs lexicaux connectés, du fait de la généralisation et de la démultiplication de leurs emplois, constituent ensemble les bases d'une métaphore iconique globale utilisée pour décrire les 
changements contemporains et de traduire les perceptions de ceux-ci. Je propose que, malgré l'instabilité, au moins apparente, de leurs sens, ce nœud lexical de tropes, un méta modèle, soit considéré comme un tout cohérent, un arrière-plan hypertextuel, un architexte même - ie " ensemble des catégories générales, ou transcendantes - types de discours, modes d'énonciation, genres littéraires, etc. - dont relève, chaque texte singulier $\aleph^{10}$. Cette acception a été spécifiée depuis la révolution digitale pour désigner une "structure hybride héritée de l'informatique, de la logique et de la linguistique " ${ }^{11}$. L'architexte est un outil d'ingénierie textuelle qui jette un pont entre la technique et la langue et plus largement les architextes constituent un imaginaire de la communication. Ils sont la praxis « de théories communicationnelles mises en œuvres, -consciemment ou non-, par leurs concepteurs ; lesquels, situés au commencement et au commandement de l'acte d'écrire, détiennent un pouvoir certain sur la production du texte, partant sur celle du sens et de l'interprétation. $»^{12}$. Parce que le triangle conceptuel de la mobilité, de l'hybridité et de la liquidité donne à entendre et à voir de nouvelles configurations dans les rapports aux catégories d'entendement classiques du temps, de l'espace, des structures sociales et politiques, des individus et des identités, remonter les courants de langue qui l'agitent permettra, je l'espère, de proposer une nouvelle intelligibilité du monde et même, peut-être, de déboucher sur une nouvelle vision d'ensemble.

Ces métaphores de la mobilité, de l'hybridité et de la liquidité sont ainsi à la fois l'outil, l'objet et l'objectif de l'investigation pratique et théorique, ce qui permet de coupler une approche nominaliste et une perspective matérialiste. Car il est possible grâce à cette méthode de circuler plus aisément entre l'analyse de texte, l'enquête de terrain et l'élaboration théorique. Ceci afin de tenter d'établir à la fois une carte des champs de bataille et des stratégies pour la guerre sémantique en cours, dont l'enjeu est le contrôle de la langue pour la structuration des espaces symboliques déchirés entre taxinomies des acteurs étatiques et entreprises globalisées, subjectivités collectives et activités des agents non-humains.

\section{Mobilité, Hybridité, Liquidité : les mots-clés de la modernité globalisée}

Au-delà de la sociologie proprement dite, les trois notions de mobilité, d'hybridité et de liquidité sont devenues centrales dans les sciences humaines et sociales. Je propose ici un rapide survol de leurs champs sémantiques et scientifiques, d'autant plus délicat, mais aussi pertinent, il me semble, que chaque notion porte un peu des deux autres en son sein. Une après l'autre je tente de tracer, entre diverses sciences et thématiques, l'évolution de leurs sens, leurs circulations, leurs imbrications et leurs conflictualités pour essayer découvrir leurs significations les plus contemporaines si tant est qu'elles puissent être stabilisées bien longtemps. L'objectif est d'en estimer la portée et le potentiel heuristique dans les théories critiques contemporaines. Tout en tenant le fil d'une réflexion sur les difficultés de l'usage des métaphores dans les sciences et dans les dispositifs de communication en général, je tente enfin d'articuler ces concepts pour élaborer un programme d'investigation adapté à la globalisation contemporaine. 


\section{La mobilité : du point de vue sédentaire à la mise en mouvement globale}

10 La croissance des mouvements humains, en particulier migratoires, a favorisé la constitution d'une thématique considérée parfois comme un nouveau paradigme du contemporain, celui des mobilités ${ }^{13}$. La mobilité n'est pas, évidemment, sans lien avec les deux notions d'hybridité et de liquidité évoquées en tant que circulation, déplacement et accélération. Et son usage est en croissance constante ${ }^{14}$.

11 Pourtant jusqu'à la fin du XXème siècle les principales sciences sociales ont entretenu des rapports ambigus avec le concept de mobilité en raison des fondements fixistes de leur constitution (la société, l'Etat, le territoire). La sociologie s'est peu préoccupée de mobilités, sociale ou spatiale et, lorsque cela a été le cas, notamment avec les migrations de populations, elles ont été perçues d'abord comme un potentiel de perturbation et de déstabilisation ${ }^{15}$. Il en a été de même pour la géographie, concentrée sur la localisation des emplacements utiles sur des cartes et la délimitation des possessions étatiques, incapable de représenter le mouvement autrement qu'avec des lignes liant un lieu à un autre ${ }^{16}$. L'histoire s'est, elle aussi, consacrée essentiellement au processus de constitution des Etats attachés à la fixation des populations pour produire des surplus, des impôts et des hommes ${ }^{17}$, mythifiant les grandes invasions inaugurales, et achoppant sur la rareté des traces laissées par les groupes mobiles. Quelle que soit l'importance des mobilités réelles dans les sociétés et les espaces étudiés, les sciences sociales ont eu tendance à restituer des systèmes étrangement immobiles, ou, à tout le moins, insensibles en apparence aux déplacements, peu visibles, considérés comme résiduels, exceptionnels, pathologiques. Ces dernières ont longtemps perçu la mobilité du point de vue opposé de la sédentarité, comme un risque, un problème, une menace.

Cela tient au fait que la modernité étatique et marchande a œuvré avant tout à une maîtrise de l'espace, à l'émergence d'individus au sein des sociétés et à la sécurisation des échanges. La mobilité humaine moderne a d'abord été marquée essentiellement par ses caractéristiques institutionnelles, sous la forme des Traites esclavagistes, des colonisations et des déplacements de masse organisés ${ }^{18}$. A la suite de la période initiale de déplacements forcés de la Grande Transformation ${ }^{19}$ les grandes institutions de contrôle de la mobilité et de construction des réseaux ont émergé dans une double capacité à déplacer et à fixer les groupes humains en fonction de considérations économiques et politiques ${ }^{20}$. La World History et l'histoire des migrations ont montré comment les déplacements de populations depuis le XVIème siècle avaient façonné le monde actuel ${ }^{21}$. Ces dernières décennies ont vu apparaitre des travaux portant sur des mobilités et des immobilités dans le sens d'une part, de l'accélération des déplacements et des conséquences induites en matière d'identité et de sociabilité et d'autre part, des formes institutionnelles de fluidification et de contrôle croisées des réseaux et des circulations urbaines ou internationales cette fois pensées comme des ressources.

La mobilité est ainsi devenue un mot clé de la période actuelle. Et cette évidence s'est imposée aux sciences sociales. J. Urry ${ }^{22}$ a demandé à ce que la sociologie soit désormais la science de la mobilité plus que de la société et $\mathrm{M}$. Castells ${ }^{23}$ considère que l'espace des flux a pris le dessus sur l'espace des lieux. Pour le géographe D. Harvey ${ }^{24}$, la mobilité et le nouveau rapport à l'espace du capital caractérisent la période contemporaine et $\mathrm{D}$. Retaillé proclame que «l'espace est mobile $»^{25}$. La globalisation est donc en grande partie 
affaire de mobilité. En matière économique, elle se caractérise par l'augmentation des circulations de capitaux, de biens, d'hommes et d'idées et par le développement accéléré des réseaux urbains et des techniques de transports et de communication ${ }^{26}$. Les notions d'entreprise en réseau, de flux tendu et de chaîne de valeur traduisent le rapport nouveau du capital à l'espace en comparaison des modèles tayloristes et fordistes (logistique, délocalisations, spatial fix, élites globalisées hypermobiles). En termes politiques, la sophistication des institutions de contrôle des mobilités et des circulations ${ }^{27}$, longtemps sous-estimée, a fait de la maîtrise des mobilités une question clé, dorénavant au centre de l'activité des Etats, des entreprises et des structures internationales ${ }^{28}$ pour gérer la porosité des frontières, le contrôle social ${ }^{29}$ et la croissance des circulations et des extractions. Concernant sa dimension culturelle, la mobilité a explicitement changé de valeur. Les représentations qui y sont associées sont passées de la menace pour les ordres sociaux, religieux et politiques ${ }^{30}$ à une valeur de changement positif, d'injonction à, et de signe de performance ou de plaisir ${ }^{31}$. La capacité à la mobilité (parfois contractée en motilité) est devenue une grille de lecture et de classement hiérarchisante des individus et des espaces ${ }^{32}$.

Dans la période actuelle, la mobilité apparait de plus en plus comme une dimension centrale en raison de la concentration urbaine et financière du pouvoir, de l'accélération de la vitesse de circulation et du désencastrement territorial du capital fixe, des marchandises et des informations numériques, de l'avènement de la société en réseaux et de la mise en mouvement, locales comme globales, de masses croissantes d'individus (transnationalisme, économie de la migration, déplacements forcés). Toutes ces évolutions tendent à remettre en cause les capacités des Etats à contrôler leur territoire. Enfin la redécouverte des modèles économiques mobiles (bazar, colportage, économies informelles -Shuttle Trade, Poor to Poor) et des mobilités autonomes (transnationales, locales ou diasporiques ${ }^{33}$ ) s'accompagne d'un accroissement de la part en déplacement des populations actives, d'une banalisation des formes d'habitats mobiles et alternatifs ${ }^{34}$ et d'un renouvellement des capacités à l'émancipation et à la mobilisation ${ }^{35}$. La mobilité est dorénavant considérée comme le moteur d'un processus général d'une part d'hybridation des cultures, des identités et des organisations et d'autre part de liquidation des institutions, des catégories et des délimitations classiques.

\section{L'hybridité : de la monstruosité stérile à la subversion créative}

15 L'hybridité est, en biologie, le caractère d'un individu issu d'un croisement d'espèces (processus de reproduction/fécondation du vivant interspécifique associant une introgression -combinaison de caractéristiques et une hétérosis -vigueur et résistance ${ }^{36}$. Hybrida (ou ibrida) veut dire en latin "sang mêlé » avec parfois le sens de "bâtard ». Le terme est longtemps employé pour désigner par exemple le croisement d'une truie et d'un sanglier mais aussi un enfant de parents de « divers pays ou de diverses conditions». L'hybridation est étudiée notamment par Lamarck ${ }^{37}$ qui en tire comme principale conséquence que les limites entre les espèces ne sont pas aussi solides qu'imaginées. Elle est en revanche mise pour partie de côté par Darwin $^{38}$ et le darwinisme social plus particulièrement comme antinomique avec la pureté. Les politiques eugénistes et nazis en Europe et ailleurs se sont notamment focalisées sur les risques de l'hybridation perçue comme un empoisonnement d'une race pure. On trouve donc un second sens péjoratif de " mélange » ou de " monstre », un " assemblage » de pièces différentes dont le produit n'appartient à aucune catégorie. La dimension racialisée est d'abord affirmée avec l'idée 
que l'hybride (individu ou espèce), a fortiori lorsqu'il s'agit d'un être humain, serait " dégénéré » par rapport à la pureté de la forme originelle. Et, au sens figuré, qualifier un nom ou une forme culturelle d'hybride revient classiquement à suggérer qu'il n'appartient à aucun style (artistique, littéraire, architectural, linguistique, etc.) ; la connotation est là aussi durablement méprisante, et renvoie à l'idée d'un type ou d'un genre hétéroclite, composite, disparate, bâtard, voire issu d'une contamination ou d'un contresens. En un mot, l'hybride est bizarre, bigarré et extravagant.

Pourtant le terme a acquis depuis quelques décennies, en plus d'une généralisation de son usage dans les contextes du Digital et du Biological Age ${ }^{39}$, un sens de créativité, de robustesse et de spécificité. La globalisation elle-même est comparée à une hybridation général ${ }^{40}$. Elle est associée à des champs allant de l'art à la technologie, de la linguistique à la musique et la création ${ }^{41}$ (sampling, intertextualité, itérabilité, traduction) et, au-delà, de la religion ${ }^{42}$ (syncrétisme) à la culture ${ }^{43}$ (acculturation, créolisation...). C'est une notion d'usage courant aussi en Science Studies, là substantivée en Hybrides post-humains ${ }^{44}$, mais aussi dans les Public Affairs et en politiques publiques. Présente en théorie de la régulation ${ }^{45}$, la notion d'hybridation institutionnelle a été utilisée à propos du tournant néolibéral des Etats du Welfare ${ }^{46}$ que du processus d'européanisation de l'action publique ${ }^{47}$ ou encore en socioéconomie de l'Internet ${ }^{48}$.

Ce terme est devenu l'enjeu d'une guerre idéologique autour des conséquences culturelles de la globalisation. Ses usages et ses valorisations sont extrêmement divers. Par exemple l'hybridation culturelle peut être dénoncée comme l'un des effets les plus néfastes de l'uniformisation capitaliste et de soumission impériale à la globalisation. Z. Bauman considère que cette métaphore active est promue par une élite prédatrice hypermobile mondialisée ${ }^{49}$. Mais elle apparaît par exemple dans les Cultural Studies ${ }^{50}$ et les Postcolonial studies $^{51}$ et en anthropologie plus largement ${ }^{52}$, comme l'instrument ultime de subversion et d'émancipation du rapport de domination colonial (Hybridity, Mimicry, Third Spaces, indigénisation, brésilianisation...). Cette notion influence évidemment la sociologie des migrations et du multiculturalisme, non seulement à travers les questions de «métissage » mais aussi de celles de l'émancipation et de l'autonomie des sujets minoritaires ou mobiles et de l'émergence de nouveaux acteurs transnationaux non étatiques.

Dans un article paru en $2014^{53}$ nous avons en effet proposé de comprendre ce processus dialectique général d'hybridation (i-e combinaison de caractéristiques) comme une indigénisation globale en l'observant d'un double point de vue des organisations, étatiques et marchandes, et des sociétés civiles. Nous avons identifié deux schémas d'indigénisations/hybridations antagoniques mais non incompatibles, dont les rapports de force influent et influeront sur les formes de transformations politiques globales et locales dans le siècle en cours. Il y a d'une part une forme d'indigénisation institutionnelle, comprise comme l'application, avec de nombreuses boucles de rétroactions, des types de gestion coloniale à l'ensemble de la planète et des activités humaines. Cela concerne les dispositifs de gouvernementalité et de colonialité mixtes, articulant et circulant entre institutions étatiques et entreprises marchandes, univers licites et illicites, temps de guerre et temps de paix ${ }^{54}$.

Il y a d'autre part ce que M. Sahlins ${ }^{55}$ décrit comme une capacité des sociétés à indigéniser la modernité, c'est-à-dire à s'approprier les cultures hégémoniques et colonisatrices et à les détourner afin de s'en émanciper. C'est une indigénisation par le bas. Le Brésil ${ }^{56}$ tout comme la Corée du Sud (mouvement culturel dit du Hallyu -la Vague) sont 
caractéristiques de cette indigénisation culturelle contemporaine. Elle recouvre à la fois les processus d'appropriation locaux/globaux des codes culturels locaux/globaux ${ }^{57}$, et de très forts potentiels d'émancipation par la subversion politique dans les mouvements sociaux, les réseaux numériques, la création artistique, etc. L'Amérique du Sud est souvent présentée comme le laboratoire global de l'indigénisation ${ }^{58}$.

Si le concept d'hybridation est si puissant et son usage si répandu c'est qu'il est construit à partir d'une métaphore correspondant à l'âge post-raciste génétique et numérique et qu'il permet de qualifier de très nombreuses formes de processus sociaux en particulier dans les sociétés technologiques et multiculturelles. Les conditions de son emploi sont néanmoins difficiles car la combinaison des sens -le mot est en soi un enjeu politique, expose au risque de la confusion voire de renvoi à des modèles raciaux " originaux $»^{59}$. En revanche il est heuristique en ce qu'il constitue un point d'entrée et une grille de lecture dans l'architexte de la globalisation. Cette piste socio-sémantique permet de rapprocher des champs, des théories et des terrains très distants a priori et rarement pensés ensemble.

\section{La notion de liquidité : de la clarté à la liquéfaction du monde}

La notion de liquidité (déjà très complexe en chimie qui la considère comme un tiers-état incertain entre le solide et le gazeux) peut, par certains aspects, paraitre emblématique de ces écueils que rencontrent les sciences humaines et sociales pour établir des sens stabilisés des notions alors même que les termes et les images circulent entre elles. Allant des connotations les plus positives aux plus négatives (que l'on élargi ici à son champ sémantique basique -liquide, liquider, liquidation, liquéfaction et autres termes hydrauliques, maritimes, aquatiques, etc.), c'est un registre universel mais dont l'usage n'a pas cessé de s'étendre au XXème siècle ${ }^{60}$. Depuis sa signification originelle en latin ( Liquidus : qui coule, qui n'a pas de forme propre, avec un sens de clarté) elle a acquis progressivement le statut de notion et d'imagerie de base d'abord en droit ${ }^{61}$ puis en économie ${ }^{62}$.

C'est un cas de métaphorisation du discours scientifique et technique tout à fait intéressant d'un point de vue rhétorique et sémiologique. Si le terme de liquidation en droit tire son sens originel de l'idée de clarté et applique ce terme principalement aux problématiques de résolution de questions commerciales et financières (liquider une affaire : fixer le montant d'une dette ou d'une pension), le registre liquide est fondateur dans la réflexion sur les "sources du droit $»^{63}$. Il tend aussi vers l'hydraulique dans la formule de droit anglais (Tort Law) de «floodgates» (vannes, écluses) en tant que tendance dans les jugements à éviter les flux de contentieux. Il prend plutôt en économie la signification de valeur disponible et capable de changer de forme et même de prendre potentiellement toute forme de biens accessibles sur un marché. C'est à travers la circulation sanguine et l'hydrologie, images centrales de l'économie depuis S. de Gramont ${ }^{64}$, A. Turgot ${ }^{65}$, D. Humes et A. Smith ${ }^{66}$ jusqu'à J. M. Keynes ${ }^{67}$ et aux néo-libéraux, que s'associe la monnaie au fluide vital indispensable à la richesse des nations ${ }^{68}$. Mais, à l'instar des particules atomiques, il s'agit d'un état mobile de la richesse, constatable mais difficilement contrôlable et mesurable.

23 Avec la financiarisation globale de l'économie, et l'américanisation de son vocabulaire (to float a loan, cash flow...), la métaphore est devenue omniprésente en ce qu'elle décrit la capacité des acteurs institutionnels de l'économie globalisée d'acheter ou de vendre en 
rendant liquide les actifs ${ }^{69}$. Le registre plombier de la liquidité (injection, fluctuation, ponction, pression...), en raison de sa force d'évocation, s'est par ailleurs étendu à tout un ensemble de types d'opérations et d'acteurs financiers caractérisés par leur opacité et leurs fuites ou leurs manipulations (évasion fiscale, corruption -Swiss Leaks, Off Shore, Laundering...). Portée par les révolutions bancaire et numérique contemporaines (le trading algorithmique notamment) la liquidité (les liquidités) a ainsi pris le sens d'une masse immense et du perpétuel mouvement de l'essentiel de la richesse rendue virtuelle dans une tuyauterie géante. Elle est incarnée par l'activité des officines financières comme les Dark Pool et traduite par des analogies hydrauliques multiples dans le vocabulaire économique ${ }^{70}$ (Trickle down effect, Pipeline, Stream, Leaks, ...). La liquidité est de plus devenu le cadre de compréhension intuitive des termes clés de l'économie et du travail contemporain autour des notions d'écoulement et de circulation d'une part et d'autre part de flexibilité et de fluidité. La liquidité économique déploie ainsi un registre sémantique qui va de la liberté, la transparence et la clarté à l'opacité et l'imprévisibilité jusqu'à la prévarication et la destruction ${ }^{71}$. Cette vulgarisation imagée du registre d'emploi du terme se retrouve ainsi dans le champ guerrier et mafieux avec la notion de liquidation d'ennemis, dans son sens anglo-saxon d'annihilation, mais aussi de la liquidation de pays, à l'occasion de la crise financière de la Grèce par exemple, contrainte à écouler à vil prix les biens publics pour rembourser ses créanciers.

Nous n'abordons ici que rapidement les lexiques liquides (hydrologie, hydraulique, plomberie, marine et cataclysmes marins, etc.) qui sont très présents dans les sciences migratoires $^{72}$ depuis F. Ratzel au moins (vagues, flux, courants...) et les sciences numériques (navigation, Open-Source, Big Torrent, Amazon, streaming...), dans des registres très proches ${ }^{73}$. Nous pouvons néanmoins noter la présence de ce type de vocabulaire et de formules dans le langage technique et scientifique de base mais aussi dans les parlers populaire, médiatique et politique. Dans les deux champs les connotations sont polarisables synthétiquement entre des acceptions positives et négatives mais partagées par l'ensemble des acteurs. Pour les migrations le caractère liquide ressort bien souvent d'un registre menaçant (crue, déferlante, raz-de-marée) mais les théories diasporiques ont mis en évidence le caractère potentiellement vertueux de la liquidité des migrations dans le monde contemporain qui en font des acteurs économiques fluides et réactifs. Dans le cas du numérique et des circulations dans le cyberespace le rapprochement avec la liquidité conserve certes une dimension inquiétante ${ }^{74}$ (par exemple avec la figure du pirate informatique évoluant dans le Dark Net ou le Deep Web-sombre et profond) mais recèle des acceptions plus positives (liberté de communication horizontale, fluidité des codes...) voire utopiques concrets (hackerspaces, démocratie liquide et transparence, partis pirates... $)^{75}$.

En sociologie et en anthropologie, le registre métaphorique maritime, hydraulique et aquatique est commun ${ }^{76}$. Cela vient compléter la tendance classique à l'usage littéraire et scientifique de ce type de métaphores pour exprimer le changement de forme et le mouvement, voire la création au sens large ${ }^{77}$. Mais l'on doit à Z. Bauman ${ }^{78}$ sa substantivation en liquide dans une acception plutôt négative pour rendre compte des effets de la globalisation et de l'individualisation ${ }^{79}$. Selon Bauman, repris par des théoriciens de la mobilité ${ }^{80}$ mais aussi du numérique et du politique, la massification des mouvements humains (touristes versus vagabonds) et leurs reconfigurations rapides constituent l'un des éléments principaux de la période de mutation actuelle tant en 
matière d'identités individuelles et collectives que de démantèlement / recomposition des institutions classiques, solides, de la modernité (trinité Etat, Nation, Territoire).

Les registres de la métaphore y associent le sens d'incertitude et de peur, de flexibilité et de fluidité. La globalisation est un schibboleth, un mot de passe pour le gué. La synthèse que propose $\mathrm{Z}$. Bauman distingue une première modernité solide, débutant vraiment avec la révolution industrielle, marquée par la mise en place d'institutions durables, par l'arraisonnement de la nature et des éléments, par le contrôle des territoires et la relocation des populations. La deuxième, liquide, est caractérisée la fluidification des cadres sociaux et politiques sous l'influence de la mondialisation économique, la dissolution des liens sociaux par la consommation et l'individualisation, la liquidation des institutions et des résistances politiques par la circulation du capital et des marchandises. La liquidité est ainsi fragilité (frailty), impuissance et liquéfaction, vacuité et impermanence ${ }^{81}$.

27 Comme pour l'hybridité, la liquidité opère sur un registre sémantique si large et ambivalent que les emplois textuels scientifiques dans les SHS sont délicats. En tant qu'image centrale dans différents champs centraux de la période actuelle mais dans des sens idéaux-typiques distants (clair, en circulation, opaque, fécond, inexorable, mortel...) elle doit être considérée comme un nœud signifiant pour les sciences humaines et sociales. Je pense en effet comme S. Sassen ${ }^{82}$ que le rapprochement des différentes problématiques liées par exemple aux transports, aux mobilités et à la gestion des liquides (des fluides), et au premier rang duquel l'eau, est un fil potentiellement riche pour les approches pluridisciplinaires de la globalisation extractiviste.

\section{Conclusion : vers un architexte de la globalisation}

Les notions de mobilité, d'hybridité et de liquidité constituent donc, selon moi, un nouveau triptyque conceptuel susceptible de rendre compte des mutations contemporaines de la globalisation. Il est en passe de remplacer le triangle classique comtien Milieu/Race/Temps (dont il hérite du concept de réseau ${ }^{83}$ ) qui a dominé les représentations avec les registres métaphoriques organicistes depuis la révolution humaniste et industrielle ${ }^{84}$. Le mobile, subversif, longtemps rendu invisible dans les mondes de la sédentarité, est devenu central, les flux prenant le pas sur les lieux, les passants bousculant les habitants, l'espace et le temps, rétréci ou accéléré, devenant mouvants et instables, incertains. L'hybride, lui, bouscule la race et les gènes, qui ont structuré toute la pensée moderne, bien qu'en en étant issu directement. Il incarne la différence et contamine. Il questionne les identités, les classifications et les frontières jusqu'au post-humanisme. Enchevêtrée dans un nœud linguistique post moderne avec ces deux notions avec lesquels elle partage de nombreux caractères, celle de liquide est dotée d'une force démultipliée, envahissante, à la fois par son caractère intrinsèquement informel et par sa grande capacité universelle à faire image (de la vie, de la mort, du temps, du pouvoir...).

Ces trois termes partagent une dimension non-humaine et sont, comme faits et comme récits, conjointement révélateurs de l'idée d'un emballement, d'une perte de contrôle des hommes sur leur destin quand la Modernité promettait à perpétuité la maitrise et l'arraisonnement de l'histoire et de la nature par l'Etat et le Progrès. En tant que modèles métaphoriques ils sont fondamentalement ambivalents, mais ils véhiculent des images simples et transposables sans effort, alors que propres à des processus d'une grande 
complexités ${ }^{85}$. Autrement dit ces qualités leurs donnent une infinie capacité à être appropriées comme expériences à la portée de tous et en tant que modes d'accès à des phénomènes incommensurables. Ils sont adaptés au contexte d'une pensée de la catastrophe et du développement d'une peur généralisée dans un monde non seulement post-moderne et post-humain mais explicitement sous la menace de plus en plus précise d'un remplacement par les clones et les cyborgs, les machines et les algorithmes, ravagé et remplacé par des espèces invasives, les eaux montantes et l'accélération infinie. Ils correspondent aussi à un temps de transition numérique et politique provoquant menaces de surveillance et domination généralisées et émergence de nouveaux outils d'échanges et de résistance. Si les schémas narratifs et esthétiques qui leurs sont associés, sont pour partie captés par des organisations dotées de forts capitaux rhétoriques à des fins de propagande marchandes et consumériste, ils sont susceptibles dans le même temps d'être retournés et réappropriés par les subjectivités collectives des multitudes.

Ils sont ainsi à même de constituer les bases d'un architexte de la globalisation, à la fois imaginaire culturel, guide du sens et grille d'interprétation, capable de structurer un nouveau programme empirique global d'investigation des et par les SHS (en lien avec sciences numériques et de la vie), pour penser les métaphores comme à la fois des instruments de pouvoir et des langues des résistances et d'émancipations individuelle et collective $^{86}$, à une époque de transformation accélérée et de fluidification, voire de dissolution des cadres sociaux et des institutions ${ }^{87}$. Enfin, les trois termes, et c'est le plus important sans doute, sont susceptibles de fournir les bases conceptuelles et pratiques d'un programme culturel et politique communément partageable notamment pour la défense des mobiles, des altérités et des Communs, par la société civile mondiale face aux acteurs étatiques et marchands ${ }^{88}$. Par son caractère autonome et incontrôlable la mobilité y apportera la furtivité et la subversion en mouvement face aux solides frontières. Par sa force de transformation et d'imitation, l'hybridité transmettra la ruse telle celle de la Mètis d'Ulysse contre les identités clôturées. Par sa puissance horizontale inexorable d'infiltration et de répétition la liquidité fournira l'arme fatale à opposer à la verticalité étatique et à la marchandisation des flux.

\section{NOTES}

1. James P. \& Steger M. B., "A Genealogy of 'Globalization': The Career of a Concept", Globalizations, 11.4, 2014, pp. 417-434 http://www.tandfonline.com/doi/ abs/10.1080/14747731.2014.951186

2. Leclerc R., Sociologie de la mondialisation, Paris, La Découverte, 2013.

3. Brown R., Clefs pour une poétique de la sociologie, Arles, Actes Sud, 1989 ; Passeron J.-C., Le raisonnement sociologique. L'espace non-poppérien du raisonnement naturel, Paris, Nathan, 1991 ; Thomas H., "Vulnérabilité, fragilité, précarité, résilience, etc. De l'usage et de la traduction de notions éponges en sciences de l'homme et de la vie. », Recueil Alexandries, Editions Terra-Hn, 2012, http://www.reseau-terra.eu/article697.htm 
4. Bernardot M. \& Thomas H., « Notes sur l'hybridité », Asylon(s), 13, 2014, Trans-concepts : lexique théorique du contemporain, http://www.reseau-terra.eu/article1327.html ; Bernardot M., «Remettre les pauvres en mouvement : Enclosures, invisibilisation et émancipations » in Cousin G. \& Loiseau G. (dir.), Actualité de l'habitat temporaire, Marseille, Terra-Hn éditions, 2015, http://www.shs.terra-hn-editions.org/Collection/?-Actualite-de1-habitat-temporaire-1; Bernardot M., " Topiques de la mobilité comme subversion. Temps, figures, héros ", in Mesini B. (dir.), Mobil Hom(m)es, formes d'habitats et mode d'habiter la mobilité, La Tour d'Aigues, eéditions de l'aube, 2016.

5. Thomas H., Les Vulnérables. La démocratie contre les pauvres, Marseille, Editions Terra-Hn, 2012, http://reseau-terra.eu/article933.html

6. Eco U., De Superman au Surhomme, Paris, Éditions Grasset \& Fasquelle, 1978 ; Eco U., Les Limites de l'interprétation, Paris, Éditions Grasset, 1992.

7. Derrida J., La dissémination, Paris, Le Seuil, 1972.

8. Camporesi P., La sève de la vie, Paris, Le Promeneur, 1990.

9. Marcus G. E., "Ethnography in/of the World System: The Emergence of Multi-situed Ethnography", Annual Review of Anthropology, 24, 1995, pp.95-117, http:// isites.harvard.edu/fs/docs/icb.topic809839.files/Readings/marcus_ethnography\%

20_in_of_world_system.pdf; Tsing A., "The Global Situation", Cultural Anthropology, 45.3, 2000, p. 327-360, http://www.jstor.org/stable/656606

10. Genette G., Introduction à l'architexte, Paris, Le Seuil, 1979, pp. 87-88.

11. Jeanneret Y. \& Souchier E., "Pour une poétique des écrits d'écran ", Xoana, 6, 1999, pp. 97-107.

12. Ablabi D. \& Ducard D., (dir.), Vocabulaire des études sémiotiques et sémiologiques, Paris, Besançon, Honoré Champion, Presses universitaires de Franche-Comté, 2009, pp. 158-159.

13. Cresswell T. \& Merriman P. (ed.), Geographies of Mobilities: Practices, Spaces, Subjects, Farnham, Ashgate, 2011, pp. 239-25 ; Faist T., "The mobility turn: a new paradigm for the social sciences?", Ethnic and Racial Studies, 36.11, 2013, pp. 1637-1646 ; Kaufman V., «La motilité : une notion clé pour revisiter l'urbain ? ", in Bassand M., Kaufmann V., Joye D., (dir.), Enjeux de la sociologie urbaine, Lausanne, Presses polytechniques et universitaires romandes, 2001, pp. 87-102 ; Le Marchand A., Enclaves nomades, Habitat et travail mobiles, Marseille, Terra-Hn éditions, 2011, https://www.reseau-terra.eu/article1192.html ; Retaillé D., « De l'horizon borné à l'horizon ouvert», Bulletin de la Société Géographique de Liège, 62.1, 2014, http://popups.ulg.ac.be/0770-7576/index.php?id=109 ; Urry J., Sociologie des mobilités, une nouvelle frontière de la sociologie ?, Paris, A. Colin, 2010 ; Sheppard E., "The Spaces and Times of Globalization: Place, Scale, Networks, and Positionality", Economic Geography, 78.3, 2002, pp. 307-330, http://www.jstor.org/stable/4140812

14. Voir (https://books.google.com/ngrams/graph?content=mobility)

15. Gallez C. \& Kaufmann V., "Aux racines de la mobilité en sciences sociales. Contribution au cadre d'analyse socio-historique de la mobilité urbaine», in Flonneau, M., Guigueno V., De l'histoire des transports à l'histoire de la mobilité. Rennes, Presses Universitaires de Rennes, 2009, pp. 41-55.

16. Retaillé D., «Du paradigme sahélien du lieu à l'espace (mondial) mobile», L'information géographique, 75.1, 2011, pp. 71-85.

17. Scott J.C., «La montagne et la liberté ou Pourquoi les civilisations ne savent pas grimper », Critique internationale, 2001, pp. 85-104. 
18. Cresswell $T$., "The vagrant/vagabond: The curious career of a mobile subject", Geographies of Mobilities: Practices, Spaces, Subjects, Cresswell T. \& Merriman P. (ed.), Farnham, Ashgate, 2011, pp. 239-254 ; Feldman D., "Migrants, immigrants and welfare from the Old Poor Law to the Welfare State", Transactions of the Royal Historical Society, 13, 2003, pp. 79-104 ; Walters W., "Deportation, Expulsion and the International Police of Aliens", Citizenship Studies, 6.3, 2002, pp. 265-292.

19. Polanyi K., La Grande transformation. Aux origines politiques et économiques de notre temps, Paris, Gallimard, 1986.

20. Foucault M., «Il faut défendre la société », Cours au Collège de France $(1976,1977)$, Paris, H.E. Gallimard, Seuil, 1997 ; Foucault M., Sécurité, territoire, population. Cours au Collège de France (1977-1978). Paris, Hautes études, Gallimard, Seuil, 2004 ; Thomas H., La production des exclus, Paris, Puf, 1997.

21. Rediker M., Outlaws of the Atlantic: Sailors, Pirates, and Motley Crews in the Age of Sail, Boston, Beacon Press, 2014 ; Samaddar R., "Returning to the History of the Late Nineteenth and Early Twentieth century Immigration", Asylon(s), 10, Défaire le cadre national des savoirs, 2014, http://www.reseau-terra.eu/article1323.html

22. Urry J., op. cit., 2010.

23. Castells M., L'ère de l'information. La société en réseaux, vol. 1, Paris, Fayard, 1998.

24. Harvey D., Géographie et capital : Vers un matérialisme historico-géographique, Paris, Syllepse, 2010.

25. Retaillé D., 2014, op. cit.

26. Sassen S., La Globalisation. Une sociologie, Paris, Gallimard, 2009.

27. Papastergiadis N., The Turbulence of Migration: Globalization, Deterritoralization, Hybridity, Cambridge, Polity Press, 2000.

28. Bernardot M., Captures, Marseille, Editions Terra-HN, 2012, https://www.reseau-terra.eu/ article1263.html

29. Papadopoulos D., Stephenson N., Tsianos V., Escape Routes: Control and Subversion in the Twenty-first Century, Londres, Ann Arbor Mi., Pluto Press, 2008.

30. Creswell T., 2011, op. cit.

31. Elliott A. \& Urry J., Mobile Lives, London, Routledge, 2010.

32. Bauman Z., Le coût humain de la mondialisation, Paris, Hachette, 1999 ; Faist T., 2013, op. cit. ; Kauffmann, 2001, op. cit.

33. Portes A., " La mondialisation par le bas. L'émergence des communautés transnationales ", Actes de la recherche en sciences sociales, 129.1, 1999, pp. 15-25 ; Tarrius A., La mondialisation par le bas. Les nouveaux nomades de l'économie souterraine, Paris, Balland, 2002 ; Waldinger R. D., "Immigrant Transnationalism", Sociopedia.isa, pp. 1-13, 2011, http://works.bepress.com/roger_waldinger/42

34. Bernardot M., (dir.), Habitats non ordinaires et espace-temps de la mobilité, Marseille, Terra-Hn éditions, 2014, https://www.reseau-terra.eu/article1341.html

35. Castells M., Networks of outrage and hope: Social movements in the internet age, Cambridge, John Wiley \& Sons, 2013.

36. Auffray C. \& Jacquard A., Dictionnaire de la biologie, Paris, Flammarion, 1998, pp. 144-145.

37. Lamarck J.-B., Philosophie zoologique, Paris, Flammarion, 1994 (1809).

38. Darwin C., L'origine des espèces, Paris, Seuil, 2013 (1859). 
39. Voir https://books.google.com/ngrams/graph?content=hybridity

40. Pieterse J. N., "Globalization as Hybridization ", in Featherstone M., Lash S. and Robertson R. (eds), Global Modernities, Londres, Sage, 1995, pp. 45-68.

41. Harris J., Critical Perspectives on Contemporary Painting. Hybridity, Hegemony, Historicism, Liverpool, Liverpool University Press and Tale, 2003.

42. Bastide R., Les religions africaines au Brésil, Paris, PUF, 1960.

43. Canclini N. G, Hybrid Cultures: Strategies for Entering and Leaving Modernity, Minneapolis, University of Minnesota Press, 1995 ; Canclini N. G., «Cultures hybrides et stratégies communicationnelles », Hermès, La Revue, 3, 2000, pp. 71-81.

44. Haraway D. J., Manifeste cyborg et autres essais : sciences, fictions, féminismes, Paris, Exils éditeurs, 2007 ; Latour B., Nous n'avons jamais été modernes, Paris, La Découverte, 1994.

45. Boyer R., «Les analyses historiques comparatives du changement institutionnel : quels enseignements pour la théorie de la régulation? » Annuels, 2003, pp. 167-203.

46. Jobert B., Le tournant néo-libéral en Europe, Paris, L'Harmattan, 1994.

47. Muller P., "L'européanisation des politiques publiques ", Politiques et management public, 15. 1, 1997, pp. 3-9.

48. Lessig, L., Remix: Making art and commerce thrive in the hybrid economy, New York, The Penguin Press, 2008, http://www.bloomsburyacademic.com/view/ Remix_9781849662505/chapter-ba-9781849662505-chapter-0008.xml

49. Bauman Z., Le présent liquide, Paris, Seuil, 2007 ; voir aussi Taguieff P.-A., " Une nouvelle illusion théorique dans les sciences sociales : la globalisation comme 'hybridation' ou 'métissage culturel' ", 2009, http://luette.free.fr/spip/spip.php? article272

50. Canclini N. G., 2000, op. cit. ; Gilroy P., L'Atlantique noir. Modernité et double conscience, Paris, Éditions Kargo, 2003 ; Hall S., Identités et cultures. Politiques des Cultural Studies, Paris, Editions Amsterdam, 2007 ; Hannerz U., Transnational Connections : Culture, People, Places, Londres, Routledge, 1996.

51. Bhabha H. K., Les lieux de la culture : une théorie postcoloniale, Paris, Payot, 2007 ; Said E. W., Culture et impérialisme, Paris, Fayard/Le Monde diplomatique, 2000.

52. Appadurai A., Après le colonialisme. Les conséquences culturelles de la globalisation, Paris, Payot, 2005.

53. Bernardot \& Thomas, 2014, op. cit.

54. Astorga L., \& Shirk, D. A., 2010, "Drug trafficking organizations and counter-drug strategies in the US-Mexican context", http://escholarship.org/uc/item/8j647429 ; Bayart J. F., Ellis S., \& Hibou, B., La criminalisation de l'Etat en Afrique, Bruxelles, Complexe, 1997 ; Bernardot M., "Permanence des camps et renouveau de la théorisation sur le confinement des étrangers ", in Makaremi C., Kobelinski C. (dir.), Enfermés dehors. Le confinement des étrangers. Perspectives de terrain, Marseille, Terra-Hn éditions 2008, pp. 105-121 ; Golberg D. T., The Racial State, Oxford, Cambridge, Blackwell, 2002 ; Golberg D. T., The Racial State, Oxford, Cambridge, Blackwell, 2002 ; Perera S.," 'What Is a Camp?' Borderphobias - the Politics of Insecurity Post-9/11", Borderlands e-journal, 1.1, 2002, http://www.borderlandsejournal.adelaide.edu.au/vol1no1_2002/perera_camp.html.

55. Sahlins M. D., How "Natives" Think. About Captain Cook, for Example, Chicago-London, University of Chicago Press, 1995. 
56. Rolnik S., "Avoiding False Problems: Politics of the Fluid, Hybrid, and Flexible", e-flux journal, 25, 2011, http://www.e-flux.com/journal/25/67892/avoiding-false-problemspolitics-of-the-fluid-hybrid-and-flexible/

57. Castells M., Communication et pouvoir, Paris, Maison des Sciences de l'Homme, 2013.

58. Linera A. G., Pour une politique de l'égalité, Paris, Les prairies ordinaires, 2008 ; Sanchez R. et Moulier-Boutang Y., « Laboratoire Amérique latine : hybridation, interdépendance et pouvoir constituant », Multitudes, 4. 35, 2008, pp. 34-39.

59. Chivallon C., «La diaspora noire des Amériques, Réflexions sur le modèle de l'hybridité de Paul Gilroy » L'Homme, 1-161, 2002, pp. 51-73 ; Young R. J. C., Colonial Desire: Hybridity in Theory, Culture and Race, Londres, Routledge, 1995.

60. Voir https://books.google.com/ngrams/graph?content=liquidity

61. Belley J.G., (dir.), Le droit soluble. Contributions québécoises à l'étude de l'intentionnalité, Paris, LJDJ, 1996 ; Vogliotti M., " De la pureté à l'hybridation : pour un dépassement de la modernité juridique ", Revue interdisciplinaire d'études juridiques, 1.62, , 2009, pp. 107-124, http://www.cairn.info/revue-interdisciplinaire-d-etudes-juridiques-2009-1-page-107.htm

62. Baré J.-F., "Images de la finance ", L'Homme, 31.119, 1991, pp. 23-40, http:// www.persee.fr/doc/hom_0439-4216_1991_num_31_119_369401; Rollo A., «Les métaphores dans le lexique économique: modèles culturels en œuvre», in P. Ligas, P. Frassi, (dir.), Lexiques Identités Cultures, Verona, Qui Edit, 2012, pp. 153-175. http:// www.dea.univr.it/documenti/Avviso/all/all252956.doc

63. Ost F., « Penser le droit aujourd'hui. L'exemple de la théorie des sources », in L. Lalonde et S. Bernatchez, La norme juridique reformatée, Les Editions Revue de droit, Sherbrooke, 2016, pp. 3-97.

64. Gramont S., Le Denier royal, Genève, Slatkine Érudition, 2014, (1620).

65. Turgot A., article Foire, in Guvres de, texte établi par Eugène Daire, Guillaumin, 1844, pp. 291-298.

66. Pour ces deux auteurs se reporter à Rosanvallon P., Le libéralisme économique. Histoire de l'idée de marché, Paris, Seuil, 1989.

67. Keynes J. M., Théorie générale de l'emploi, de l'intérêt et de la monnaie, Paris, Payot, 2017.

68. Pasanek B. \& Polillo S., (dir.), Beyond Liquidity: The Metaphor of Money in Financial Crisis, Londres, Routledge, 2013.

69. Ho K., Liquidated : an ethnography of Wall Street, Durham, Duke University Press, 2009.

70. Gillespie R., "From circulation to asymmetric flow: On metaphors and global capitalism", Journal of Cultural Economy, 6. 2, 2013. pp. 200-216.

71. Neyrat F., Clinamen. Flux, absolu et loi spirale, Alfortville, Ere éditions, 2011.

72. Bernardot M., «Petit traité de navigation dans la langue migratoire », Multitudes, 3.64, 2016, pp. 63-70, https://www.cairn.info/revue-multitudes-2016-3-page-63.htm

73. Bernardot M., «Plongée dans les métaphores liquides de la langue numérique. Exploration socio-sémiologique du territoire digital et de la globalisation ", Netcom, à paraître en 2018.

74. Benito-Ruiz E., "Infoxication 2.0", in Thomas M., Handbook of Research on Web 2.0 and Second Language Learning, Pennsylvania, IGI-InfoSci, 2009, pp. 60-79, http:// storage.vuzit.com/public/a7l/Draft2ok_Ruiz.pdf ; Cardon D., A quoi rêvent les algorithmes. Nos vies à l'heure des Big Data, Paris, Seuil, 2015 ; Poirrier P., " "Marée montante" » ou tsunami ? L'avenir du livre à l'heure du numérique», Le livre électronique au présent. Pratiques de lecture, de prescription et de médiations, 2015, pp. 123-126, https:// 
halshs.archives-ouvertes.fr/halshs-01174872/document; Sadin E., La Vie algorithmique : critique de la raison numérique, Paris, L'Echappée, 2015.

75. Area M. \& Pessoa T., "From solid to liquid: New Literacies to the cultural changes of Web 2.0", Comunicar, 19.38, 2012, pp. 13-20, https://www.revistacomunicar.com/pdf/ preprint/38/En-01-PRE-12378.pdf ; Auray N., «Pirates en réseau: détournement, prédation et exigence de justice » Esprit, 7, 2009, pp. 168-179, http://ses-perso.telecomparistech.fr/auray/2009AurayEsprit.pdf ; Béguin-Verbrugge A., « Métaphores et intégration sociale des technologies nouvelles ", Communication et langages, 141.1, 2004, pp. 83-93, http://www.persee.fr/doc/colan_0336-1500_2004_num_141_1_3292 ; Broca S., Utopie du logiciel libre. Du bricolage informatique à la réinvention sociale, Neuvy-enChampagne, Editions le passager clandestin, 2013, http://lepassagerclandestin.fr/ fileadmin/assets/catalog/essais/

Utopie_logiciel_libre__Broca__Le_passager_clandestin.pdf

76. Carse A., «Water», Cultural Anthropology, 2010, http://www.culanth.org/ curated_collections/10-water ; Helmreich S., "Nature/Culture/Seawater", American Anthropologist, 113, 2011, pp. 132-144, http://onlinelibrary.wiley.com/doi/10.1111/ j.1548-1433.2010.01311.x/full ; Krause F \& Strang V., "Living Water: the powers and politics of a vital substance", Worldviews: Global Religions, Culture, and Ecology, 17.2, 2013, pp. 95-102 ; Mosse D., The rule of water: statecraft, ecology and collective action in South India, Oxford, Oxford University Press, 2003.

77. Bachelard G., L'eau et les rêves : essai sur l'imagination de la matière, Paris, J. Corti, 1942.

78. Voir parmi les nombreux textes concernés Bauman Z., L'Amour liquide, De la fragilité des liens entre les hommes, Rodez, Éditions du Rouergue, 2005 ; Bauman Z., La Vie liquide, Rodez, Éditions du Rouergue, 2006.

79. Davis M., Freedom and Consumerism. A Critique of Zygmunt Bauman's Sociology, Aldershot, Ashgate, 2012 ; Jacobsen M. H. \& Poder P. (ed.), The Sociology of Zygmunt Bauman. Challenges and Critique, Aldershot, Ashgate, 2008.

80. Papastergiadis N., 2000, op. cit. ; Elliot A. \& Urry J., 2010, op. cit.

81. Jacobsen M. H. \& Marshman S., "Bauman on Metaphors. A Harbinger Hybrid Sociology", in Jacobsen M. H. \& Poder P. (ed.), The Sociology of Zygmunt Bauman. Challenges and Critique, Aldershot, Ashgate, 2008, pp. 19-40.

82. Sassen S., Expulsions: Brutality and complexity in the global economy, Cambridge, Harvard University Press, 2014.

83. Musso P., Critique des réseaux, Paris, PUF, 2003.

84. Canguilhem G., La connaissance de la vie, Paris, Vrin,1989, (1952).

85. Deleuze G., Cinéma : L'image-mouvement, Paris, Minuit, 1983.

86. Lizcano E., Metáforas que nos piensan. Sobre ciencia, democracia y otras poderosas ficciones, Madrid, Traficantes de Sueños, 2006.

87. Lewkowicz I., Pensar sin Estado, la subjectivida en la era de la fluidez, Buenos Aires, Paidos, 2004.

88. Beck U., Pouvoir et contre-pouvoir à l'heure de la globalisation, Paris, Flammarion, 2003. 
INDEX

Mots-clés : mobilité, hybridité, mondialisation, globalisation, architexte

\section{AUTEUR}

MARC BERNARDOT

Professeur des Universités, Aix-Marseille 